\title{
Itraconazole inhibits invasion and migration of pancreatic cancer cells by suppressing TGF-ß/SMAD2/3 signaling
}

\author{
KE CHEN* ${ }^{*}$, LIANG CHENG* ${ }^{*}$ WEIKUN QIAN, ZHENGDONG JIANG, LIANKANG SUN, YANFEI ZHAO, \\ YONGSHENG ZHOU, LIZHI ZHAO, PENGLI WANG, WANXING DUAN, QINGYONG MA and WEI YANG
}

\begin{abstract}
Department of Hepatobiliary Surgery, First Affiliated Hospital, Xi'an Jiaotong University, Xi'an, Shaanxi 710061, P.R. China
\end{abstract}
Received June 4, 2017; Accepted December 5, 2017

DOI: $10.3892 /$ or.2018.6281

\begin{abstract}
Pancreatic cancer is the fourth leading cause of cancer-associated mortality worldwide, with an overall 5 -year survival rate $<8 \%$. We studied the therapeutic effect of itraconazole (ITZ), a commonly used broad-spectrum anti-fungal agent, in the treatment of pancreatic cancer, and to reveal the underlying anticancer mechanisms. Effects of ITZ on cell proliferation, apoptosis, invasion and migration were observed by MTT assays and colony formation assays, flow cytometry, wound scratch assays and transwell assays, respectively. Western blotting and immunofluorescence were performed to investigate the effect of ITZ on the epithelial to mesenchymal transition (EMT) of pancreatic cancer cells. Recombinant transforming growth factor- $\beta$ (TGF- $\beta$ ) and TGF- $\beta$ neutralizing antibody were used to study the effect of ITZ on the TGF- $\beta /$ SMAD $2 / 3$ signaling. Transgenic engineered mice which harboring the spontaneous pancreatic cancer was applied to investigate the therapeutic role of ITZ in vivo. We report that ITZ inhibited the viability and induced apoptosis of pancreatic cancer cells. Furthermore, ITZ suppressed the invasion and migration of pancreatic cancer cells. We found that ITZ treatment was efficient in suppressing EMT and that the effect of ITZ was partially mediated by impaired TGF- $\beta$ / SMAD2/3 signaling. The role of TGF- $\beta /$ SMAD2/3 signaling in mediating the effect of ITZ was confirmed based on the results that recombinant TGF- $\beta$ induced, but the TGF- $\beta$ neutralizing antibody inhibited EMT as well as the invasion and migration of pancreatic cancer cells. Also, the anticancer effect of ITZ could be partially reversed by recombinant
\end{abstract}

Correspondence to: Dr Wei Yang or Dr Qingyong Ma, Department of Hepatobiliary Surgery, First Affiliated Hospital, Xi'an Jiaotong University, 277 West Yanta Road, Xi'an, Shaanxi 710061, P.R. China E-mail: drbodyang@163.com

E-mail: qyma56@xjtu.edu.cn

*Contributed equally

Key words: itraconazole, epithelial to mesenchymal transition, transforming growth factor- $\beta$, pancreatic cancer, invasion and migration
TGF- $\beta$. Furthermore, treatment with ITZ suppressed growth of tumor in vivo. Taken together, we suggest that ITZ may potentially serve as a new chemotherapeutic agent for the treatment of pancreatic cancer.

\section{Introduction}

Pancreatic cancer, which is dominated by pancreatic ductal adenocarcinoma (PDAC), is currently the fourth leading cause of cancer-related mortality in developed countries, with a 5 -year survival rate $<8 \%$ (1). The initiation and progression of pancreatic cancer are always associated with non-specific symptoms. At diagnosis, most patients have developed to an advanced stage with local invasion and distant metastasis that preclude surgical resection of pancreatic cancer due to the lack of early diagnostic procedures (2). More than $80 \%$ of patients miss the opportunity for complete surgical eradication, and even for those who undergo surgery, the 5-year survival rate is still $25 \%$ (3). Gemcitabine has been regarded as the mainstay of chemotherapy for pancreatic cancer. In recent years, gemcitabine combined with FOLFIRINOX (folinic acid, 5-fluorouracil, irinotecan and oxaliplatin) or albumin-bound paclitaxel has been introduced and has shown improved therapeutic efficacy compared to gemcitabine monotherapy at the cost of serious adverse effects $(4,5)$.

Epithelial-to-mesenchymal transition (EMT) has been considered as a major driver of the initiation and progression of pancreatic cancer (6). The process of EMT is associated with elevated expression of $\mathrm{N}$-cadherin and vimentin as well as decreased expression of E-cadherin (7). The EMT process could be triggered by a number of transcriptional factors, including Snail and Slug, ZEB1, ZEB2 and Twist $(6,8)$. A previous study showed that deletion of Zeb1, one of the EMT transcriptional factors, reduced the stemness, tumorigenic and colonization capacities of cancer cells; the deletion of Zeb1 suppressed the grading, invasion and distant metastasis of pancreatic cancer in vivo (9). Further research revealed that the transcriptional factor Snail- or Twist-induced EMT program is of great importance in mediating the chemoresistance of pancreatic cancer to gemcitabine (10), which is in accordance with previous evidence that EMT is implicated in stemness and treatment resistance (6).

Members of the transforming growth factor- $\beta$ (TGF- $\beta$ ) family play important roles in the growth, differentiation and apoptosis of cells, and they exert their functions during 
the process of embryonic development (11). Previous studies have revealed that pancreatic cancer expresses high levels of TGF- $\beta$, and it correlates with poor prognosis $(12,13)$. Smad2 and Smad3, two members of the Smad family, mediate the signal transduction of TGF- $\beta$. Binding of TGF- $\beta$ with transmembrane serine-threonine kinase receptors leads to the phosphorylation and activation of downstream Smad2 and Smad3, which then translocate to the nucleus to control the transcription of target genes (11).

Itraconazole (ITZ) is a type of triazole anti-fungal agent that is widely applied clinically in the treatment and prevention of fungal infections. Recently, ITZ has been found to have great potential in the treatment of several types of cancers (14-17). A non-comparative, randomized, phase II study showed that high-dose ITZ exhibited anticancer properties in patients with metastatic castration-resistant prostate cancer (18). Another phase II study that enrolled 23 patients with progressive nonsquamous non-small cell lung cancer found that ITZ combined with pemetrexed achieved longer progression-free survival and significantly prolonged overall survival compared to treatment with pemetrexed alone (19). In addition, ITZ also showed therapeutic efficacy in basal cell carcinoma by reducing cell proliferation and tumor size (20). In addition, numerous preclinical studies have been conducted to investigate the therapeutic effect of ITZ in other types of cancers, including ovarian cancer (21), endometrial cancer (22), gastrointestinal cancer (23) and bladder cancer (17).

However, whether ITZ has antitumor effects on pancreatic cancer cells and the underlying mechanisms remain to be elucidated. In the present study, we investigated the effects of ITZ on the biological behavior of pancreatic cancer cells and the potential underlying molecular mechanisms.

\section{Materials and methods}

Cell culture and reagents. We purchased human MiaPaCa-2, Panc-1, and BxPC-3 tumor cells from the Chinese Academy of Sciences Cell Bank of Type Culture Collection (CBTCCCAS, Shanghai, China). The MiaPaCa-2 and Panc-1 cell lines were cultured in Dulbecco's modified Eagle's medium (DMEM) with $10 \%$ fetal bovine serum (FBS) (Hyclone, Logan, UT, USA), and BxPC-3 was cultured in RPMI-1640 supplemented with $10 \%$ FBS and $1 \%$ penicillin-streptomycin. The pancreatic cancer cell lines were cultured under standard conditions at $37^{\circ} \mathrm{C}$ with a $5 \% \mathrm{CO}_{2}$ atmosphere. The antibodies against E-cadherin, $\mathrm{N}$-cadherin, and vimentin were purchased from Cell Signaling Technology (Danvers, MA, USA); the antibody against $\beta$-actin was obtained from Sigma (St. Louis, MO, USA); and the antibodies against TGF- $\beta$ and p-SMAD2/3 were obtained from Abcam (Cambridge, MA, USA). ITZ was initially dissolved in DMSO (dimethyl sulfoxide) at a stock concentration of $2 \mathrm{mM}$. Working dilutions of ITZ were made in culture medium immediately before use.

Cell viability assays. The viability of pancreatic cancer cells was measured using 3-(4,5-dimethyl-2-thiazolyl)2,5-diphenyl-2-H-tetrazolium bromide (MTT) (Sigma) assays. Panc-1, BxPC-3 and MiaPaCa-2 cells were seeded onto 96-well plates at a density of 5,000 cells per well and incubated overnight in $10 \%$ FBS medium. After treatment with ITZ for 24 ,
48 and $72 \mathrm{~h}$ at $37^{\circ} \mathrm{C}$ and $5 \% \mathrm{CO}_{2}$, the inhibition rate of the pancreatic cancer cells was assessed. Briefly, $20 \mu \mathrm{l}$ of MTT solution $(5 \mathrm{mg} / \mathrm{ml}$ in distilled water) was added to each well, and the cells were incubated for $4 \mathrm{~h}$ at $37^{\circ} \mathrm{C}$ for the absorption of MTT; then, the medium was removed and replaced by $200 \mu \mathrm{l}$ of DMSO, and the optical density (OD) was measured at $490 \mathrm{~nm}$ on a multifunctional microplate reader (POLARstar OPTIMA; BMG, Offenburg, Germany).

Colony formation assays. Panc-1 and BxPC-3 cells were seeded onto $35-\mathrm{mm}$ petri dishes (1,000 cells per dish) and allowed to adhere overnight. Then, cells were treated with different concentrations of ITZ and further cultured for 2 weeks to allow colonies to form. At the indicated time point, colonies were fixed with $4 \%$ paraformaldehyde for $2 \mathrm{~h}$, stained with $0.1 \%$ crystal violet solution and rinsed. The colonies $>0.5 \mathrm{~mm}$ in diameter were counted using a microscope (Nikon Eclipse Ti-S; Nikon Instruments, Inc., Tokyo, Japan) at a magnification, $\mathrm{x} 400$.

Apoptosis assays. We assessed apoptosis of Panc-1 and BxPC-3 cells using an Annexin V-FITC/PI apoptosis detection kit (Beyotime Institute of Biotechnology, Shanghai, China) according to the manufacturer's instructions. Pancreatic cancer cells $\left(10^{5}\right.$ cells/well) were seeded onto 6-well plates and pretreated with FBS-free DMEM or RPMI-1640 for $8 \mathrm{~h}$; then, the medium was replaced with fresh medium containing varying concentrations of ITZ. A total of $48 \mathrm{~h}$ later, the cancer cells were trypsinized, washed with PBS and then stained with Annexin $\mathrm{V}$ and propidium iodide in the dark. The percentage of apoptotic cells was quantified by flow cytometry.

Western blot assays. Whole-cell lysates of Panc-1 and BxPC-3 cells were prepared using RIPA buffer (Beyotime, Guangzhou, China) according to the manufacturer's instructions. Protein concentration was determined using a BCA protein assay kit (Pierce, Rockford, IL, USA). The protein lysates were resolved on a $10 \%$ polyacrylamide gel with a $5 \%$ stacking gel. The proteins were subsequently blotted onto polyvinylidene difluoride (PVDF) membranes. The membranes were blocked for $2 \mathrm{~h}$ in TBS containing $0.1 \%$ (vol/vol) Tween-20 and 10\% (wt/vol) non-fat dry milk powder and then incubated with primary antibodies overnight at $4^{\circ} \mathrm{C}$. Following incubation with secondary HRP-coupled antibodies for $2 \mathrm{~h}$ at room temperature, the membranes were washed with $0.1 \%$ TBS-Tween-20, and the immunocomplexes were detected using the enhanced chemiluminescence (ECL) kit and a Molecular Imager ChemiDoc XRS system (Bio-Rad Laboratories, Hercules, CA, USA). $\beta$-actin was used as an internal loading control.

Immunofluorescence analysis. The cells were fixed in $4 \%$ formaldehyde diluted in phosphate-buffered saline (PBS) for 20 min. Following permeabilization with $0.3 \%$ Triton X-100, the cells were treated with blocking buffer (5\% BSA in PBS) for $1 \mathrm{~h}$ and then incubated with a primary antibody at $4^{\circ} \mathrm{C}$ overnight. Green conjugated secondary antibodies from Jackson ImmunoResearch Laboratories (West Grove, PA, USA) were applied at room temperature, and the nuclei were stained with 4'-6-diamidino-2-phenylindole (DAPI). Images were pseudocolored using a Zeiss Instruments confocal microscope. 
A

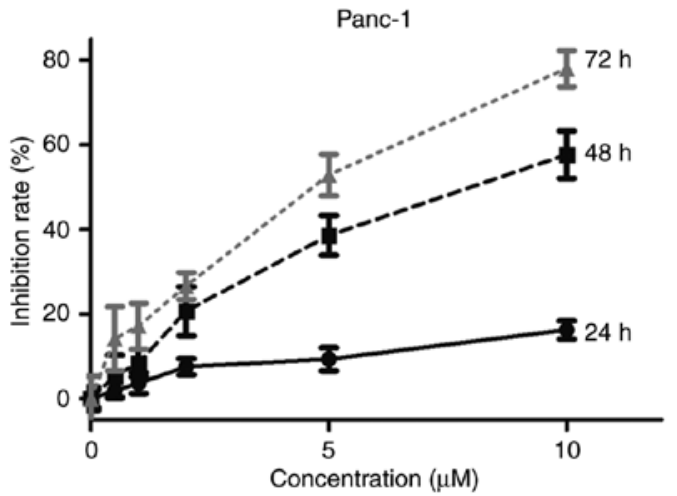

B

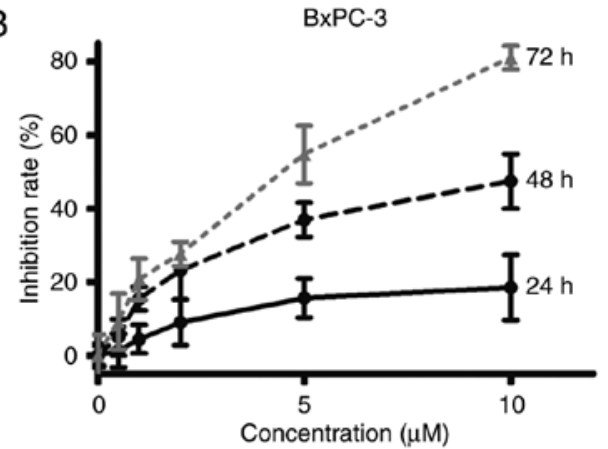

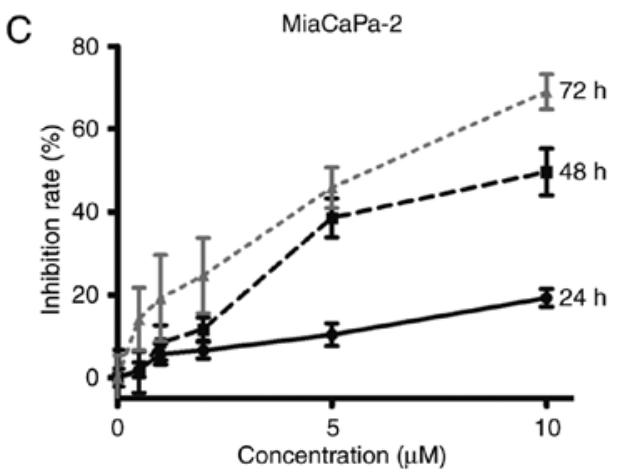

Figure 1. ITZ inhibits the viability of pancreatic cancer cells in a concentration- and time-dependent manner. Panc-1 (A), BxPC-3 (B), and MiaPaCa-2 (C) cells were treated with increasing concentrations of ITZ $(0,0.5,1,2,5$ and $10 \mu \mathrm{M})$ for 24,48 and $72 \mathrm{~h}$ to analyze the inhibition ratio for cancer cell viability. ITZ, itraconazole.

Cell invasion assays. Matrigel invasion assays were performed as previously described to assess the invasive viability of pancreatic cancer cells (24). In brief, the upper chambers of the wells were coated with Matrigel (BD Biosciences, Franklin Lakes, NJ, USA). The tumor cells $\left(10^{5}\right)$ were suspended in serum-free DMEM and seeded onto the upper chamber. The cells were allowed to invade toward the lower chamber, which contained DMEM with $10 \%$ FBS, for $48 \mathrm{~h}$ and were then fixed with $4 \%$ formaldehyde, followed by the removal of non-invasive cells in the upper chamber with a cotton swab. The invading cells were stained with $0.1 \%$ crystal violet. The number of invaded cells was quantified by counting the stained cells under a microscope.

Wound healing assays. The migration ability of the cancer cells was assessed by wound-healing assays. The cancer cells were pretreated as indicated, and then, the cells were scratched using a $10-\mu 1$ pipette tip. Next, the cancer cells were washed 3 times with PBS and cultured in serum-starved medium. Images were obtained under a microscope (Nikon Instruments, Inc.).

In vivo study. LSL-Kras ${ }^{\mathrm{G} 12 \mathrm{D} /+} ; \mathrm{p} 53^{\mathrm{H} / /+}$; Pdx1-Cre (KPC) mice were used to study the therapeutic effect of ITZ in vivo. The breeding of KPC mice was achieved by crossing the Pdx1-Cre mice with LSL-Kras ${ }^{\mathrm{G} 12 \mathrm{D}}$ mice and $\mathrm{p} 53^{\mathrm{fl} / \mathrm{fl}}$ mice (purchased from the Nanjing Biomedical Research Institute of Nanjing University, Nanjing, China). All mice were housed under pathogen-free conditions and had free access to water and food. All experimental protocols were approved by the Ethics Committee of the First Affiliated Hospital of Medical College, Xi'an Jiaotong University (Xi'an, China).
Statistical analysis. Each experiment was performed at least three times. The data are presented as the mean \pm standard deviation. Comparisons between groups were analyzed by Student's t-test using SPSS (version 15.0; SPSS, Inc., Chicago, IL, USA). P-values $<0.05$ were considered to be statistically significant.

\section{Results}

ITZ inhibits the viability of pancreatic cancer cells in a concentration-dependent manner. We first investigated the effect of ITZ on the viability of pancreatic cancer cells. Pancreatic cancer cell lines Panc-1, BxPC-3 and MiaPaCa-2 were treated with increasing concentrations of $\operatorname{ITZ}(0,0.5,1,2$, 5 and $10 \mu \mathrm{M})$. The viability of pancreatic cancer cell lines was assessed by MTT assays at the indicated time points $(24,48$ and $72 \mathrm{~h}$ ). Our results showed that ITZ inhibited the viability of pancreatic cancer cell lines in a dose- and time-dependent manner (Fig. 1). We observed that ITZ at a concentration of $2 \mu \mathrm{M}$ began to suppress the viability of pancreatic cancer, and $5 \mu \mathrm{M}$ ITZ showed a sufficient effect. A previous study that involved thirty healthy men revealed that successive intake of ITZ every $12 \mathrm{~h}$ for 14 days produced a steady plasma concentration of $2.67 \mu \mathrm{M}(25)$. According to the results of the clinical study and the MTT assay, the concentrations of 2 and $5 \mu \mathrm{M}$ were chosen for further experiments.

ITZ inhibits colony formation and induces apoptosis in pancreatic cancer cells. To further investigate the therapeutic effect of ITZ in pancreatic cancer, we set out to detect the effect of ITZ on the clone formation capability of Panc-1 and BxPC-3 cell 
A

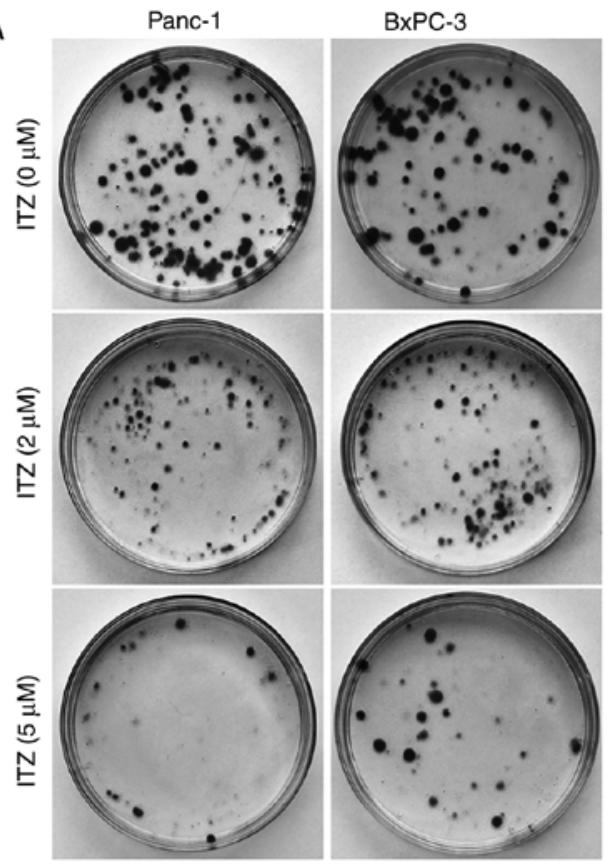

B
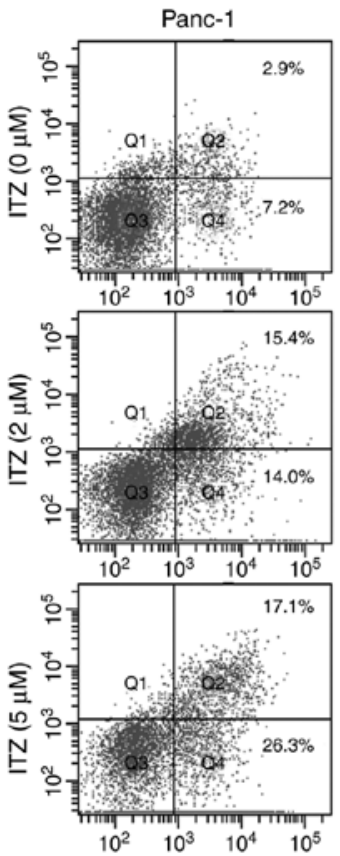
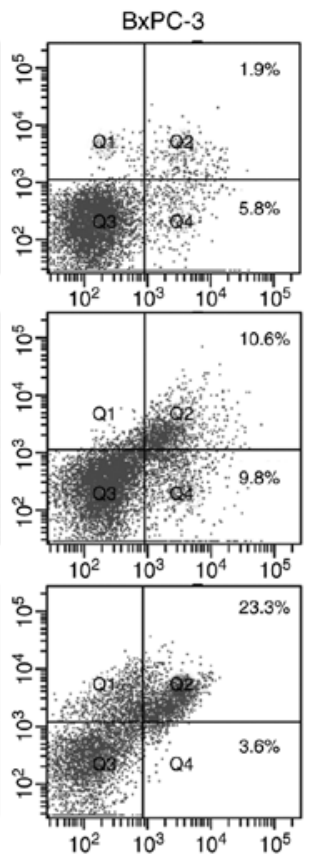
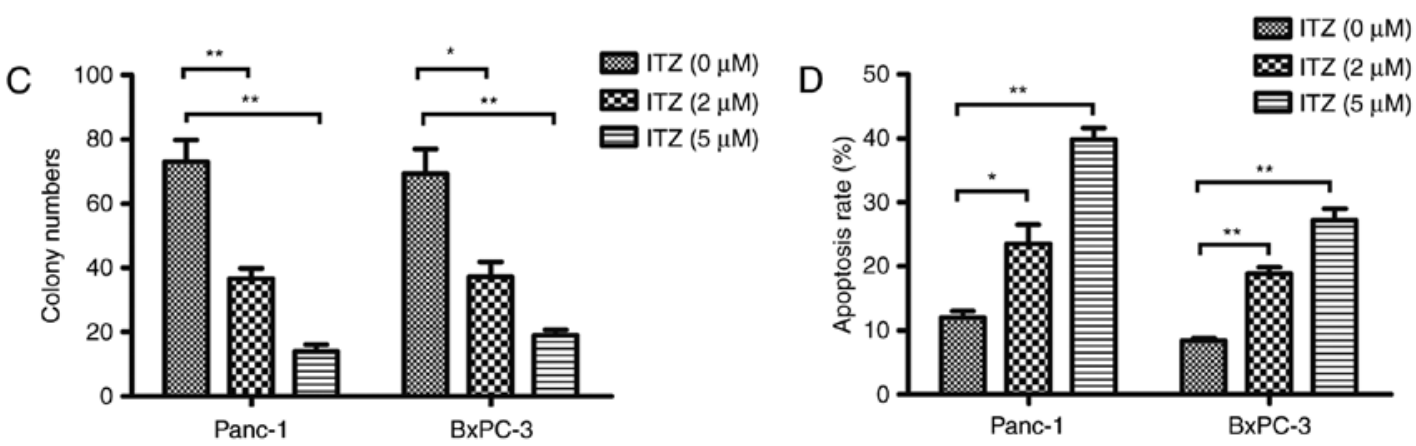

Figure 2. ITZ inhibits colony formation and induces apoptosis in pancreatic cancer cells. (A) The effect of ITZ (0, 2 and $5 \mu \mathrm{M})$ on the colony-formation ability of Panc-1 and BxPC-3 cells. Images are representative of three independent experiments. (B) The effects of ITZ on apoptosis in pancreatic cancer cells were measured by flow cytometry. (C) Quantification of the colonies formed by pancreatic cancer cells treated with different concentrations of ITZ. (D) Quantification of the apoptosis rate in cancer cells that were treated as indicated. ITZ, itraconazole. ${ }^{*} \mathrm{P}<0.05,{ }^{* *} \mathrm{P}<0.01$.

lines. Panc-1 and BxPC-3 cells were seeded onto 35-mm Petri dishes and allowed to adhere overnight; then, the cells were treated with 2 or $5 \mu \mathrm{M}$ ITZ for $48 \mathrm{~h}$. Cancer cells were cultured for two weeks, and the number of colonies were calculated. As shown in Fig. 2A, we found that ITZ at a concentration of $2 \mu \mathrm{M}$ significantly suppressed colony formation in the Panc-1 and BxPC-3 cell lines. In the dishes that were treated with $5 \mu \mathrm{M}$ of ITZ, few colonies were observed (Fig. 2C).

Next, flow cytometric analyses were performed to evaluate whether ITZ could induce apoptosis in pancreatic cancer cells. Panc-1 and BxPC-3 cells were pretreated with FBS-free DMEM or FBS-free RPMI-1640 for $12 \mathrm{~h}$ and then treated with 2 or $5 \mu \mathrm{M} \mathrm{ITZ} \mathrm{for} 48 \mathrm{~h}$. We found that compared to the vehicle-treated cells, cells treated with ITZ showed an increased percentage of apoptotic cells (Fig. 2B and D). These results revealed the role of ITZ in inhibiting colony formation and inducing pancreatic cancer cell apoptosis.

ITZ inhibits the invasion and migration of pancreatic cancer cells. Invasion and metastasis are important characteristics of pancreatic cancer, and most patients with pancreatic cancer have already developed to advanced stages with complications involving distant metastasis at the time of diagnosis (5). Thus, we explored the effect of ITZ on the invasion and migration of pancreatic cancer cells. After starvation in FBS-free DMEM or FBS-free RPMI-1640 for $12 \mathrm{~h}$, Panc-1 and BxPC-3 cells were treated with $2 \mu \mathrm{M}$ or $5 \mu \mathrm{M}$ ITZ for $48 \mathrm{~h}$. The wound healing assays were performed under serum-free conditions to evaluate the migration of cancer cells. We found that the migration abilities of Panc-1 and BxPC-3 cells were impaired by ITZ intervention compared to vehicle-treated cells (Fig. 3A and B).

To investigate the invasive ability of cancer cells after ITZ intervention, Matrigel invasion assays were conducted. We found that ITZ at the concentration of $2 \mu \mathrm{M}$ significantly inhibited the invasion of Panc-1 cells; at the concentration of $5 \mu \mathrm{M}$, the invasive abilities of Panc-1 and BxPC-3 were both suppressed (Fig. 3C and D). These findings suggest that ITZ inhibits the invasion and migration capacities of pancreatic cancer cells in vitro.

ITZ inhibits EMT and TGF- $\beta / S M A D 2 / 3$ signaling in pancreatic cancer cells. EMT is a complicated program that plays an important role in the invasion and metastasis of cancer $(26,27)$. 
A
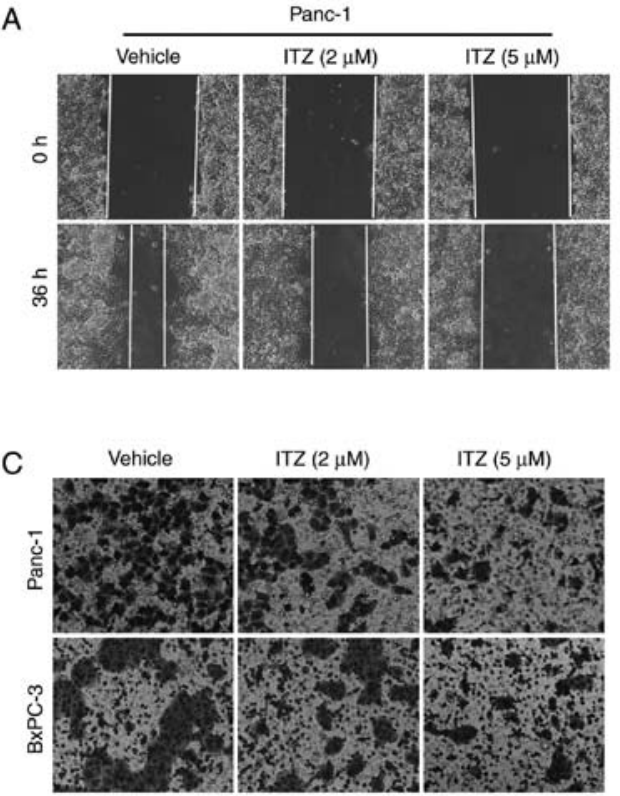

B
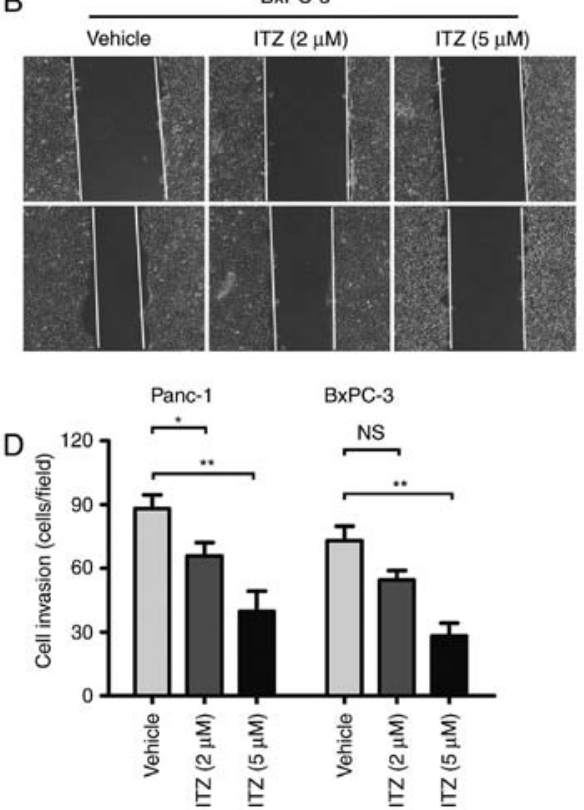

Figure 3. ITZ inhibits the migration and invasion of pancreatic cancer cells. (A) Wound healing assays were performed to detect the migration ability of Panc-1 cells. Images were acquired at 0 and $36 \mathrm{~h}$ at a magnification, x100. (B) Representative images of the wound healing assays performed with BxPC-3 cells. (C) The effects of ITZ on the invasion ability of Panc-1 and BxPC-3 cells were evaluated by Matrigel invasion assays. Images are representative of three independent experiments. (D) Quantification of the invaded cells that were treated as indicated. ITZ, itraconazole. NS, not significant; ${ }^{*} \mathrm{P}<0.05$, ${ }^{* *} \mathrm{P}<0.01$.

A

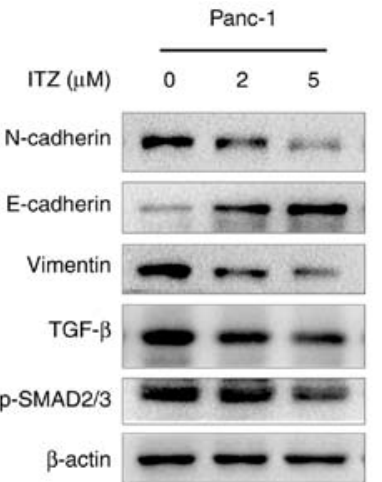

B

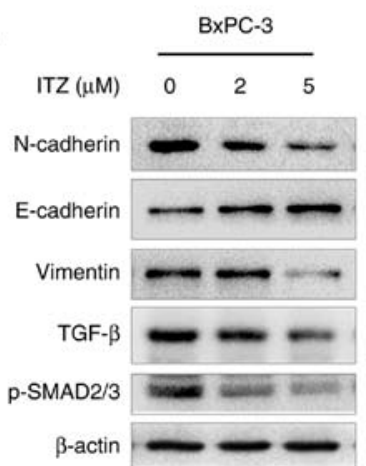

C
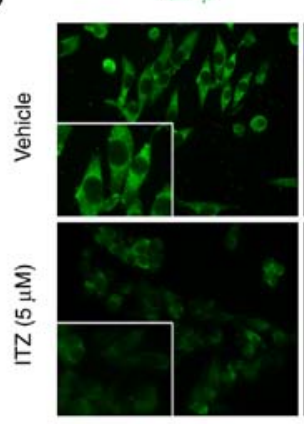

D
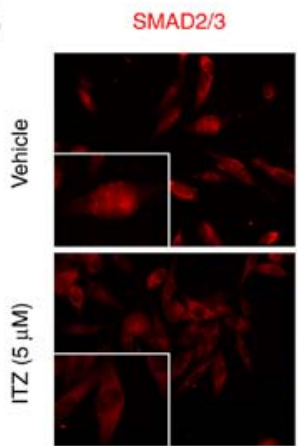

DAPI
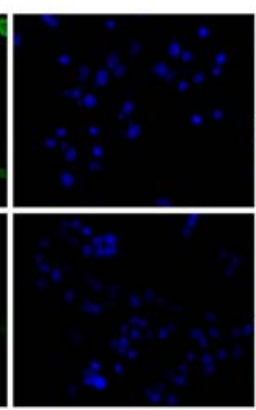

DAPI

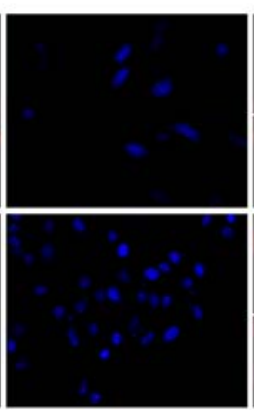

Merge

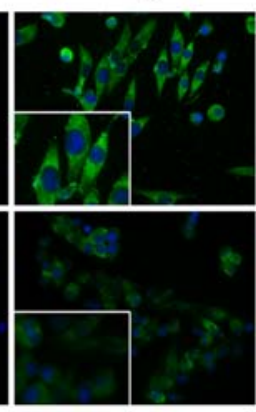

Merge

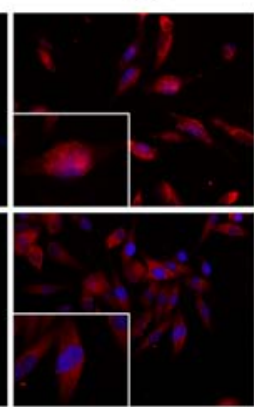

Figure 4. ITZ inhibits EMT and suppresses the activity of the TGF- $\beta / S M A D 2 / 3$ signaling pathway. (A) Western blot assays were performed to evaluate the effect of ITZ on the EMT markers N-cadherin, E-cadherin and vimentin as well as the members of the TGF- $\beta /$ SMAD2/3 signaling pathway TGF- $\beta$ and p-SMAD2/3 in Panc-1 cells. (B) Western blot assays showing the effect of ITZ on the expression of EMT markers and TGF- $\beta /$ SMAD2/3 signaling molecules in BxPC-3 cells. (C) Immunofluorescence assays showing that ITZ treatment decreases the fluorescence intensity of TGF- $\beta$. (D) Immunofluorescence assays showing that ITZ decreases the accumulation of p-SMAD2/3 in the nucleus of pancreatic cancer cells. ITZ, itraconazole. EMT, epithelial to mesenchymal transition. TGF- $\beta$, transforming growth factor- $\beta$.

We then investigated whether ITZ could affect the EMT process. We found that in Panc- 1 cells, ITZ treatment ( 2 or $5 \mu \mathrm{M})$ decreased the expression of the mesenchymal markers,
$\mathrm{N}$-cadherin and vimentin. However, expression of the epithelial marker, E-cadherin, was elevated (Fig. 4A). A similar effect was also observed regarding EMT in BxPC-3 cells (Fig. 4B). 

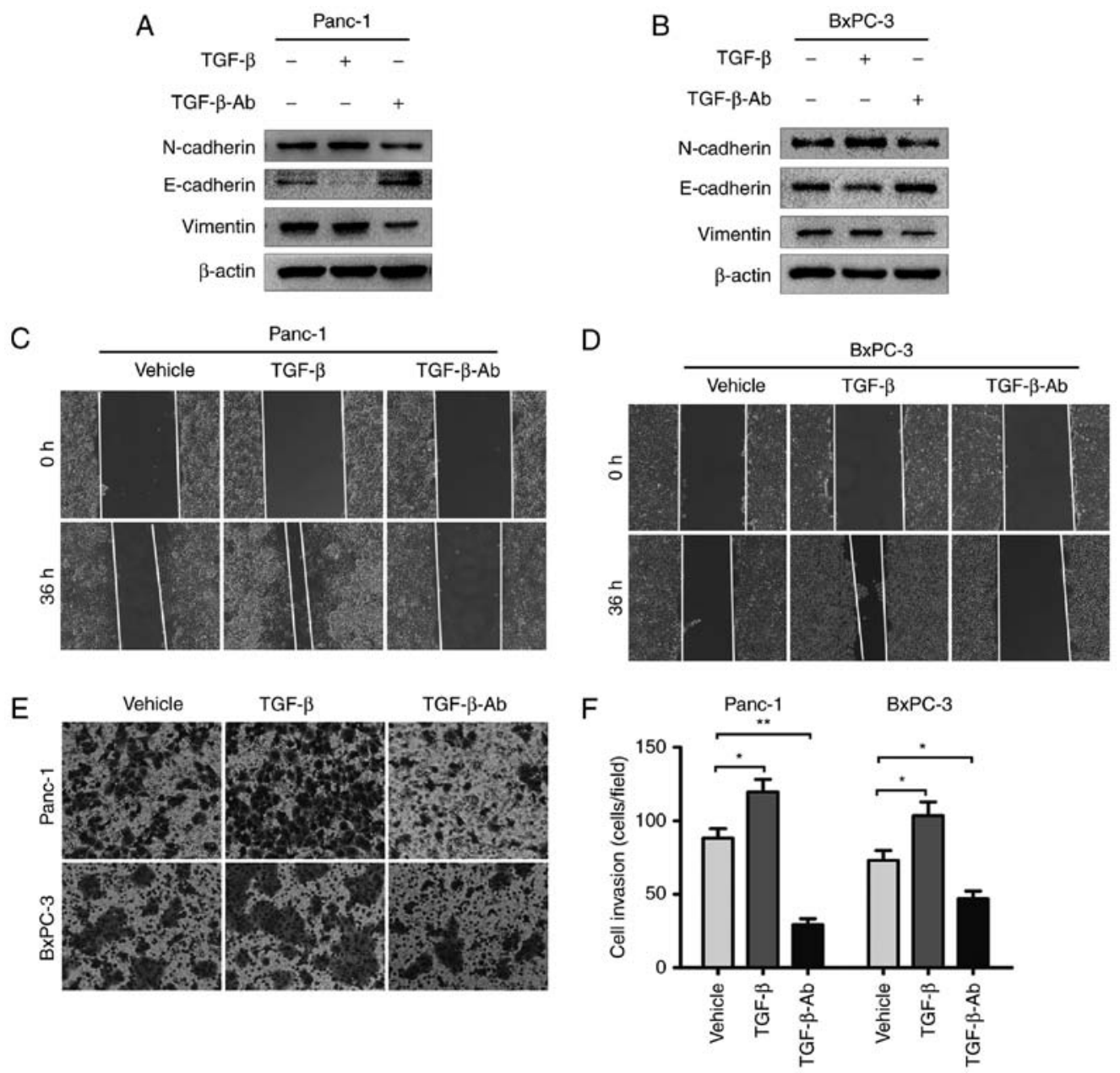

Figure 5. Recombinant TGF- $\beta$ induces EMT, invasion and migration in pancreatic cancer cells. (A and B) The expression of EMT markers N-cadherin, E-cadherin and vimentin was assessed in Panc-1 (A) and BxPC-3 cells (B) by western blot assays after treatment with recombinant TGF- $\beta$ or TGF- $\beta$ neutralizing antibody (TGF- $\beta-\mathrm{Ab}$ ) for $48 \mathrm{~h}$. (C and D) Wound healing assays showing the migration abilities of Panc-1 (C) and BxPC-3 (D) cells that were pretreated with recombinant TGF- $\beta$ or TGF- $\beta-A b$, and the images were visualized after 0 and $36 \mathrm{~h}$ at a magnification, $\mathrm{x} 40$. (E) Representative images showing the invasive abilities of pancreatic cancer cells that were treated as indicated. (F) Quantification of the invaded cells that were treated as indicated. ITZ, itraconazole. EMT, epithelial to mesenchymal transition. TGF- $\beta$, transforming growth factor- $\beta$. ${ }^{*} \mathrm{P}<0.05,{ }^{* * *} \mathrm{P}<0.01$.

Given previous evidence that TGF- $\beta$ is involved in EMT as well as the invasion and migration of cancer cells $(28,29)$, we set out to investigate the effect of ITZ on TGF- $3 /$ SMAD2/3 signaling. Western blotting was performed to detect the expression of TGF- $\beta$ and $p-S M A D 2 / 3$. As expected, we observed high expression of TGF- $\beta$ and $p$-SMAD2/3 in Panc- 1 and BxPC-1 cells. However, compared to vehicle-treated cells, the expression of TGF- $\beta$ was downregulated after ITZ treatment. Additionally, the level of p-SMAD2/3 was also decreased (Fig. 4A and B). Binding of TGF- $\beta$ with its membrane-bound receptor leads to phosphorylation of the SMAD family. The latter, in turn, translocates into the nucleus and acts as transcriptional factors to exert their functions (30). Then, we conducted immunofluorescence assays to study the effect of ITZ on TGF- $\beta /$ SMAD $2 / 3$ signaling. We observed that ITZ treatment decreased the level of TGF- $\beta$ and suppressed the nuclear accumulation of SMAD2/3 (Fig. 4C and D). These data suggest that ITZ treatment inhibits the EMT process and TGF- $\beta /$ SMAD2/3 signaling in pancreatic cancer.

TGF- $\beta$ is a potent factor mediating EMT in pancreatic cancer cells. To investigate whether the effect of ITZ on EMT is mediated by impaired TGF- $\beta / \mathrm{SMAD} 2 / 3$ signaling, we reassessed the effect of TGF- $\beta /$ SMAD $2 / 3$ signaling on EMT in pancreatic cancer cells. After starvation in FBS-free medium for $8 \mathrm{~h}$, Panc- 1 and BxPC-3 cells were treated with recombinant TGF- $\beta 1(5 \mathrm{ng} / \mathrm{ml})$ for $24 \mathrm{~h}$, and then, the EMT markers were detected by western blot assays. Recombinant TGF- $\beta 1$ significantly suppressed the expression of E-cadherin and elevated the expression of $\mathrm{N}$-cadherin and vimentin in Panc-1 and BxPC-3 cells (Fig. 5A and B). The wound healing assays showed that treatment with recombinant TGF- $\beta 1$ promoted the migration of cancer cells (Fig. 5C and D). Accordingly, the Matrigel invasion assays showed that the invasive capability of cancer cells was also increased by recombinant TGF- $\beta 1$ treatment (Fig. 5E and F).

Next, we set out to observe the effect of TGF- $\beta 1$ deprivation on the invasion and migration of pancreatic cancer. TGF- $\beta 1$ neutralizing antibody was used to block the function of TGF- $\beta 1$. As expected, treatment with TGF- $\beta 1$ neutralizing antibody elevated the expression of E-cadherin and decreased the levels of $\mathrm{N}$-cadherin and vimentin compared to vehicletreated cancer cells (Fig. 5A and B). The wound healing and Matrigel invasion assays also showed that TGF- $\beta 1$ neutralizing 
$\mathbf{A}$

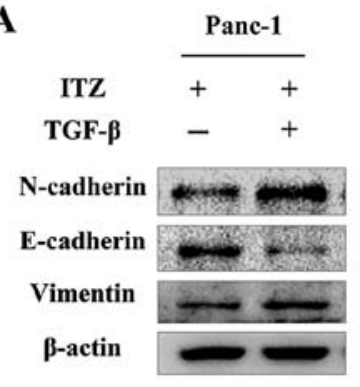

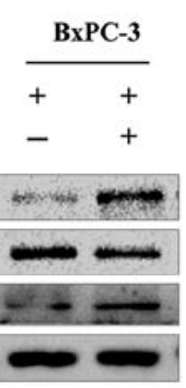
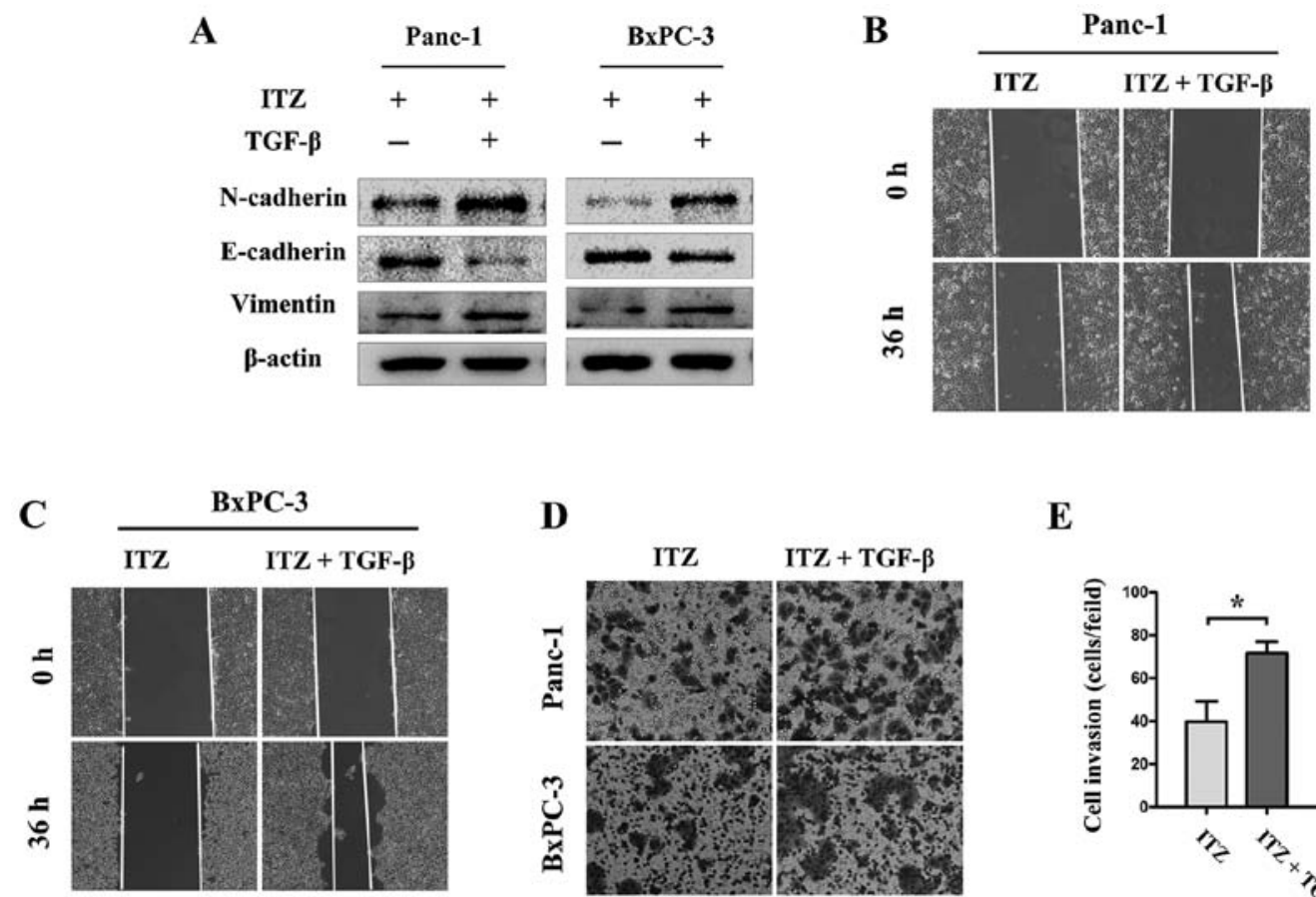

D

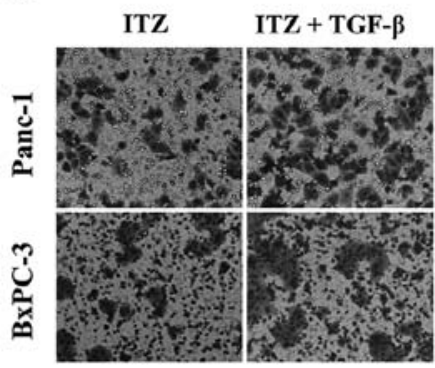

$\mathbf{E}$

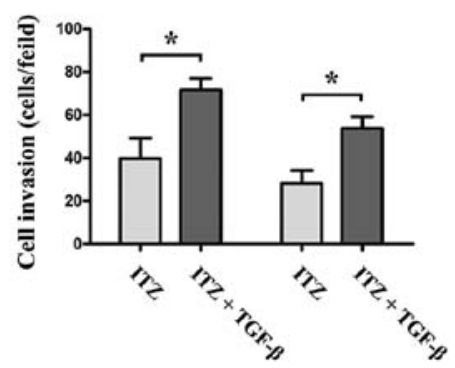

Figure 6. Recombinant TGF- $\beta$ partially reverses the effect of ITZ on pancreatic cancer cells. (A) Panc- 1 and BxPC-3 cells were pretreated with ITZ ( $5 \mu \mathrm{M})$ for $24 \mathrm{~h}$ and treated with human-derived recombinant TGF- $\beta 1(5 \mathrm{ng} / \mathrm{ml})$; then, the expression of EMT markers was assessed by western blot assays. (B and C) The wound healing assays showing that recombinant TGF- $\beta$ partially reversed the migration of Panc-1 (B) and BxPC-3 (C) cells. (D) Representative images showing that treatment with recombinant TGF- $\beta$ partially reversed the invasive capacities of pancreatic cancer cells. (E) Quantification of the invaded cells that were treated as indicated. ITZ, itraconazole. EMT, epithelial to mesenchymal transition. TGF- $\beta$, transforming growth factor- $\beta$. ${ }^{*} \mathrm{P}<0.05$.

antibody inhibited the invasion and migration of Panc-1 and BxPC-3 cells (Fig. 5C-F). We revealed that TGF- $\beta$ is a potent factor that mediates EMT, invasion and migration in pancreatic cancer cells.

TGF- $\beta / S M A D 2 / 3$ signaling is required for the effects of ITZ on pancreatic cancer. Given the results that ITZ inhibited TGF- $\beta /$ SMAD2/3 signaling as well as the invasion and migration of pancreatic cancer cells, we wondered whether TGF- $\beta /$ SMAD2/3 signaling is required for ITZ to exert its anticancer effects on pancreatic cancer. To verify our hypothesis, Panc-1 and BxPC-3 cells were pretreated with ITZ $(5 \mu \mathrm{M})$ for $24 \mathrm{~h}$, and then, the cells were treated with recombinant TGF- $\beta 1(5 \mathrm{ng} / \mathrm{ml})$. Western blot assays showed that recombinant TGF- $\beta 1$ recovered the expression of $\mathrm{N}$-cadherin and vimentin, both of which were suppressed by ITZ treatment. Accordingly, recombinant TGF- $\beta 1$ treatment inhibited the ITZ-induced expression of E-cadherin (Fig. 6A and B). In addition, the invasion and migration capabilities of Panc-1 and BxPC-3 cells were also recovered (Fig. B-E). These results showed that recombinant TGF- $\beta 1$ reversed the effect of ITZ on pancreatic cancer. We revealed that TGF- $\beta /$ SMAD2/3 signaling mediates the effects of ITZ on pancreatic cancer.

ITZ suppresses growth of pancreatic cancer in vivo. To further investigate the therapeutic effect of ITZ in vivo, we used KPC transgenic mice harboring spontaneous pancreatic cancer. Starting at 8 weeks of age, when KPC mice have developed widespread advanced pancreatic neoplasia, we treated the KPC mice with vehicle or ITZ. KPC mice were sacrificed when end-staged disease developed. We found that ITZ treatment significantly decreased the tumor weight (Fig. 7A and B). Next, immunohistochemistry was performed to detect the proliferation index, Ki67. We found that treatment with ITZ decreased the number of Ki67-positive cells (Fig. 7C and D). These results suggest the therapeutic effect of ITZ in vivo.

\section{Discussion}

Pancreatic cancer is the major cause of cancer-associated mortality, with dismal prognosis, invasion and distant metastasis as some of its dominant features. The treatment of pancreatic cancer remains a challenge because of the lack of techniques for early diagnosis and the resistance to traditional chemotherapeutic drugs (2). Therefore, new drug candidates are needed for pancreatic cancer. ITZ is a broad-spectrum anti-fungal agent that exerts its effect through the decrease of ergosterol synthesis, which is required for fungal cell membrane integrity (14). Accumulating evidence suggests that ITZ exhibits anticancer activity against various types of cancers, and therefore, we hypothesized that ITZ may be a promising potential drug in the treatment of pancreatic cancer. Here, we found that ITZ inhibits the viability of pancreatic cancer cell lines. Treatment with ITZ significantly reduced colony formation and induced apoptosis in Panc-1 and BxPC-3 cells. We noted that ITZ treatment impaired the invasion and migration abilities of pancreatic cancer cells. In addition, ITZ suppressed EMT in pancreatic cancer cells, partly through the inhibition of TGF- $\beta /$ SMAD $2 / 3$ signaling. Furthermore, ITZ suppressed the growth of pancreatic cancer in vivo. 
A

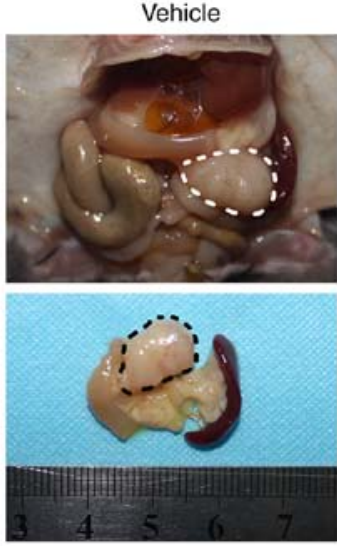

C

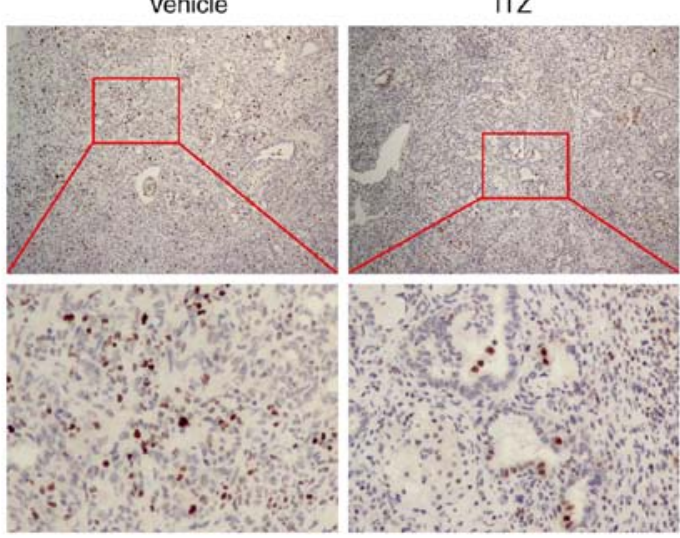

ITZ

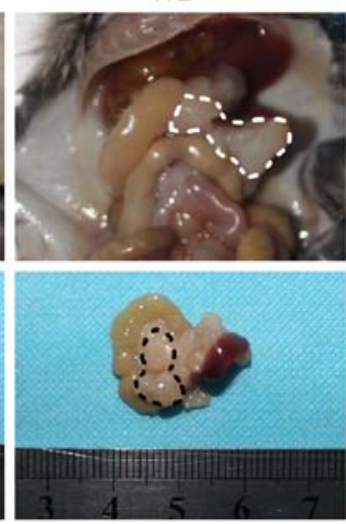

$\mathrm{B}$
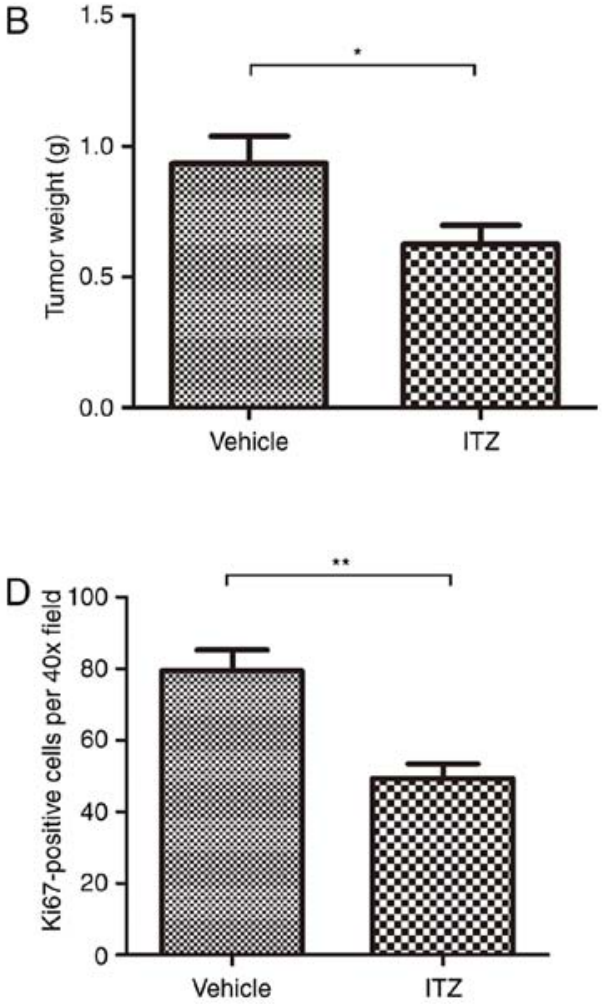

Figure 7. ITZ suppresses tumor growth in KPC mice. (A) Representative macroscopic images of pancreatic cancer in KPC mice treated with vehicle or ITZ. The white dotted line shows the pancreas and the black dotted line shows the tumor. (B) Quantification of the tumor weight in mice treated with vehicle or ITZ. (C) Immunohistochemistry showing the staining of Ki67 in the pancreatic tissues from the mice treated as indicated. (D) Quantification of the Ki67-positive cells. Scale bars, $100 \mu \mathrm{m}$. ITZ, itraconazole. ${ }^{*} \mathrm{P}<0.05,{ }^{* *} \mathrm{P}<0.01$.

TGF- $\beta /$ SMAD2/3 signaling plays vital roles in the progression of pancreatic cancer. A previous study reported that in genetically engineered mice harboring spontaneous pancreatic cancer, haploinsufficiency for the Tgfbr2 gene, which encodes the receptor for TGF- $\beta$, achieved a higher frequency of well-differentiated pancreatic cancer. In addition, haploinsufficiency for Tgfbr 2 also reduced hematogenous metastasis and suppressed the colonization of cancer cells into the liver (31), indicating the role of TGF- $\beta /$ SMAD $2 / 3$ signaling in the distant metastasis of pancreatic cancer. TGF- $\beta$ is also involved in the establishment of the immunosuppressive microenvironment of cancer, in part through inducing the synthesis and secretion of immunosuppressive cytokines, such as IL-2, IL-6 and IL-17; on the other hand, it promotes the induction of $\mathrm{CD} 8(+)$ regulatory $\mathrm{T}$ cells, which contribute to cancer progression and immune evasion $(32,33)$. TGF- $\beta$ silencing combined with immune activation induced profound tumor cell apoptosis, which is mediated by the recruitment of activated CD8-positive $\mathrm{T}$ cells and the reduced infiltration of CD11b(+) Gr-1(-) myeloid cells into the tumor (34). Here, we report that TGF- $\beta$ is sufficient to induce the invasion and migration of pancreatic cancer cells, and blocking TGF- $\beta$ significantly inhibited the invasion and migration abilities of cancer cells. We also revealed that TGF- $\beta$ is an important mediator of EMT in pancreatic cancer, and we reconfirmed the cancer-promoting role of the TGF- $\beta /$ SMAD2/3 signaling pathway in pancreatic cancer.
The anticancer activity of ITZ is mediated by various mechanisms. Aftab, et al reported that in non-small cell lung cancer, ITZ inhibited the proliferation, migration and tube formation of endothelial cells in response to the induction of the pro-angiogenic factors, including vascular endothelial growth factor (VEGF) and basic fibroblast growth factor (bFGF); additionally, oral ITZ significantly reduced tumor vascularity in vivo (35). In addition, inhibition of the Hedgehog pathway, which has been shown to contribute to the growth and progression of many types of cancers, is another important mechanism mediating the anticancer effect of ITZ (36). A recent report also revealed that ITZ-mediated Hedgehog pathway inhibition inhibited proliferation as well as induced apoptosis and autophagic cell death in breast cancer, thus inhibiting tumor growth in vivo (37). Besides the Hedgehog pathway, a recent study reported that ITZ exerts its anticancer effect by impairing a regulatory network involving the Hedgehog, Wnt, and PI3K/mTOR signaling pathways, thus inhibiting the growth of melanoma and extending the survival of mice harboring melanoma xenografts (38). Here, we found that treatment with ITZ may interfere with TGF- $\beta / S M A D 2 / 3$ signaling. We revealed a novel mechanism by which ITZ suppresses the progression of pancreatic cancer.

EMT enables cancer cells to invade the basement membranes and spread to the vasculature or lymphatic ducts to form distant metastases. Previous studies have reported that in pancreatic cancer, EMT plays context-specific roles depending 
on the different EMT-associated transcriptional factors; they reported that genetic ablation of Snail or Twist contributes to enhanced sensitivity to gemcitabine treatment without altering the incidence of systemic dissemination and metastasis, but the transcriptional factor Zeb1-induced EMT is associated with invasiveness and distant metastasis $(9,10)$. We found that ITZ is sufficient to impair EMT. Accordingly, ITZ suppressed the invasion and migration of pancreatic cancer.

For the treatment of pancreatic cancer, gemcitabine combined with FOLFIRINOX (folinic acid, 5-fluorouracil, irinotecan and oxaliplatin) or albumin-bound paclitaxel could act as the first-line chemotherapy, and the two regimens achieved an overall survival of 11.1 and 8.5 months, respectively, compared to the 6.8 months achieved by gemcitabine treatment alone $(4,5)$. However, for the patients who are resistant to the first-line regimens, a more efficient secondline regimen is required. A previous study reported that ITZ combined with other cytotoxic chemotherapeutic drugs showed a promising effect in patients with pancreatic cancer who had first received chemotherapy and had a history of progression during or after prior treatment (39). ITZ in combination with a cytotoxic regimen consisting of docetaxel, gemcitabine, and carboplatin (DGC) achieved a median overall survival of 11.4 months. Among 38 patients, the authors observed one complete and 13 partial responses (39). Here, our study provides further evidence for the therapeutic efficacy of ITZ in pancreatic cancer.

In conlusion, our results suggest that ITZ has potent anticancer properties. ITZ was able to induce apoptosis, inhibit cellular proliferation, suppress EMT and suppress the invasion and migration of pancreatic cancer cells. As a drug that has been tested for toxicity in humans and has been approved for human use, ITZ shows great potential in the treatment of pancreatic cancer.

\section{Acknowledgements}

This study was supported by grants from the National Natural Science Foundation of China (nos. 81672434, 81472248 and 81402971).

\section{References}

1. Siegel RL, Miller KD and Jemal A: Cancer Statistics, 2017. CA Cancer J Clin 67: 7-30, 2017.

2. Kleeff J, Korc M, Apte M, La Vecchia C, Johnson CD, Biankin AV, Neale RE, Tempero M, Tuveson DA, Hruban RH, et al: Pancreatic cancer. Nat Rev Dis Primers 2: 16022, 2016.

3. Kamisawa T, Wood LD, Itoi T and Takaori K: Pancreatic cancer. Lancet 388: 73-85, 2016.

4. Von Hoff DD, Ervin T, Arena FP, Chiorean EG, Infante J, Moore M, Seay T, Tjulandin SA, Ma WW, Saleh MN, et al: Increased survival in pancreatic cancer with nab-paclitaxel plus gemcitabine. N Engl J Med 369: 1691-1703, 2013.

5. Conroy T, Desseigne F, Ychou M, Bouché O, Guimbaud R, Bécouarn Y, Adenis A, Raoul JL, Gourgou-Bourgade S, de la Fouchardière C, et al; Groupe Tumeurs Digestives of Unicancer; PRODIGE Intergroup: FOLFIRINOX versus gemcitabine for metastatic pancreatic cancer. N Engl J Med 364: 1817-1825, 2011.

6. Zhou P, Li B, Liu F, Zhang M, Wang Q, Liu Y, Yao Y and Li D: The epithelial to mesenchymal transition (EMT) and cancer stem cells: Implication for treatment resistance in pancreatic cancer. Mol Cancer 16: 52, 2017.

7. Yilmaz M and Christofori G: EMT, the cytoskeleton, and cancer cell invasion. Cancer Metastasis Rev 28: 15-33, 2009.
8. Sánchez-Tilló E, Liu Y, de Barrios O, Siles L, Fanlo L, Cuatrecasas M, Darling DS, Dean DC, Castells A and Postigo A: EMT-activating transcription factors in cancer: Beyond EMT and tumor invasiveness. Cell Mol Life Sci 69: 3429-3456, 2012.

9. Krebs AM, Mitschke J, Lasierra Losada M, Schmalhofer O, Boerries M, Busch H, Boettcher M, Mougiakakos D, Reichardt W, Bronsert P, et al: The EMT-activator Zeb1 is a key factor for cell plasticity and promotes metastasis in pancreatic cancer. Nat Cell Biol 19: 518-529, 2017.

10. Zheng X, Carstens JL, Kim J, Scheible M, Kaye J, Sugimoto H, Wu CC, LeBleu VS and Kalluri R: Epithelial-to-mesenchymal transition is dispensable for metastasis but induces chemoresistance in pancreatic cancer. Nature 527: 525-530, 2015.

11. Singh P, Wig JD and Srinivasan R: The Smad family and its role in pancreatic cancer. Indian J Cancer 48: 351-360, 2011.

12. Friess H, Yamanaka Y, Büchler M, Ebert M, Beger HG, Gold LI and Korc M: Enhanced expression of transforming growth factor beta isoforms in pancreatic cancer correlates with decreased survival. Gastroenterology 105: 1846-1856, 1993.

13. Javle M, Li Y, Tan D, Dong X, Chang P, Kar S and Li D: Biomarkers of TGF- $\beta$ signaling pathway and prognosis of pancreatic cancer. PLoS One 9: e85942, 2014.

14. Pantziarka P, Sukhatme V,Bouche G, Meheus L and Sukhatme VP: Repurposing Drugs in Oncology (ReDO)-itraconazole as an anticancer agent. E Cancer Med Sci 9: 521, 2015.

15. Hu Q, Hou YC, Huang J, Fang JY and Xiong H: Itraconazole induces apoptosis and cell cycle arrest via inhibiting Hedgehog signaling in gastric cancer cells. J Exp Clin Cancer Res 36: 50, 2017.

16. Tsubamoto H, Inoue K, Sakata K, Ueda T, Takeyama R, Shibahara $\mathrm{H}$ and Sonoda T: Itraconazole inhibits AKT/ mTOR signaling and proliferation in endometrial cancer cells. Anticancer Res 37: 515-519, 2017.

17. Mamtani R, Yang YX, Scott FI, Lewis JD and Boursi B: Association of itraconazole, a Hedgehog inhibitor, and bladder Cancer. J Urol 196: 343-348, 2016.

18. Antonarakis ES, Heath EI, Smith DC, Rathkopf D, Blackford AL, Danila DC, King S, Frost A, Ajiboye AS, Zhao M, et al: Repurposing itraconazole as a treatment for advanced prostate cancer: A noncomparative randomized phase II trial in men with metastatic castration-resistant prostate cancer. Oncologist 18: 163-173, 2013.

19. Rudin CM, Brahmer JR, Juergens RA, Hann CL, Ettinger DS, Sebree R, Smith R, Aftab BT, Huang P and Liu JO: Phase 2 study of pemetrexed and itraconazole as second-line therapy for metastatic nonsquamous non-small-cell lung cancer. J Thorac Oncol 8: 619-623, 2013.

20. Kim DJ, Kim J, Spaunhurst K, Montoya J, Khodosh R, Chandra K, $\mathrm{Fu}$ T, Gilliam A, Molgo M, Beachy PA, et al: Open-label, exploratory phase II trial of oral itraconazole for the treatment of basal cell carcinoma. J Clin Oncol 32: 745-751, 2014.

21. Tsubamoto H, Sonoda T, Yamasaki $M$ and Inoue K: Impact of combination chemotherapy with itraconazole on survival of patients with refractory ovarian cancer. Anticancer Res 34: 2481-2487, 2014.

22. Inoue K, Tsubamoto H, Sakata K, Sakane R, Hao H, Hirota S, Sonoda $\mathrm{T}$ and Shibahara H: Expression of Hedgehog signals and growth inhibition by itraconazole in endometrial cancer. Anticancer Res 36: 149-153, 2016.

23. Hara M, Nagasaki T, Shiga K and Takeyama H: Suppression of cancer-associated fibroblasts and endothelial cells by itraconazole in Bevacizumab-resistant gastrointestinal cancer. Anticancer Res 36: 169-177, 2016.

24. Zhang D, Lei J, Ma J, Chen X, Sheng L, Jiang Z, Nan L, Xu Q, Duan $\mathrm{W}$, Wang $\mathrm{Z}$, et al: $\beta 2$-adrenogenic signaling regulates NNK-induced pancreatic cancer progression via upregulation of HIF-1 $\alpha$. Oncotarget 7: 17760-17772, 2016.

25. Barone JA, Moskovitz BL, Guarnieri J, Hassell AE, Colaizzi JL, Bierman RH and Jessen L: Food interaction and steady-state pharmacokinetics of itraconazole oral solution in healthy volunteers. Pharmacotherapy 18: 295-301, 1998.

26. Guo J, Wang B, Fu Z, Wei J and Lu W: Hypoxic microenvironment induces EMT and upgrades stem-like properties of gastric cancer cells. Technol Cancer Res Treat 15: 60-68, 2016.

27. Carstens JL, Lovisa S and Kalluri R: Microenvironment-dependent cues trigger miRNA-regulated feedback loop to facilitate the EMT/MET switch. J Clin Invest 124: 1458-1460, 2014.

28. Yin T, Wang C, Liu T, Zhao G and Zhou F: Implication of EMT induced by TGF-betal in pancreatic cancer. J Huazhong Univ Sci Technolog Med Sci 26: 700-702, 2006. 
29. Kong B, Michalski CW, Hong X, Valkovskaya N, Rieder S, Abiatari I, Streit S, Erkan M, Esposito I, Friess H, et al: AZGP1 is a tumor suppressor in pancreatic cancer inducing mesenchymalto-epithelial transdifferentiation by inhibiting TGF- $\beta$-mediated ERK signaling. Oncogene 29: 5146-5158, 2010.

30. Javelaud D, Alexaki VI, Dennler S, Mohammad KS, Guise TA and Mauviel A: TGF- $\beta /$ SMAD/GLI2 signaling axis in cancer progression and metastasis. Cancer Res 71: 5606-5610, 2011.

31. Zhong Y, Macgregor-Das A, Saunders T, Whittle MC, Makohon-Moore A, Kohutek ZA, Poling J, Herbst BT, Javier BM, Cope L, et al: Mutant p53 together with TGF $\beta$ signaling influence organ-specific hematogenous colonization patterns of pancreatic cancer. Clin Cancer Res 23: 1607-1620, 2017.

32. Zhou X, Mao Y, Zhu J, Meng F, Chen Q, Tao L, Li R, Fu F, Liu C, $\mathrm{Hu} \mathrm{Y}$, et al: TGF- $\beta 1$ promotes colorectal cancer immune escape by elevating B7-H3 and B7-H4 via the miR-155/miR-143 axis. Oncotarget 7: 67196-67211, 2016.

33. Wu M, Chen X, Lou J, Zhang S, Zhang X, Huang L, Sun R, Huang P, Wang F and Pan S: TGF- $\beta 1$ contributes to CD8 ${ }^{+}$Treg induction through $\mathrm{p} 38 \mathrm{MAPK}$ signaling in ovarian cancer microenvironment. Oncotarget 7: 44534-44544, 2016.

34. Ellermeier J, Wei J, Duewell P, Hoves S, Stieg MR, Adunka T, Noerenberg D, Anders HJ, Mayr D, Poeck H, et al: Therapeutic efficacy of bifunctional siRNA combining TGF- $\beta 1$ silencing with RIG-I activation in pancreatic cancer. Cancer Res 73: 1709-1720, 2013.
35. Aftab BT, Dobromilskaya I, Liu JO and Rudin CM: Itraconazole inhibits angiogenesis and tumor growth in non-small cell lung cancer. Cancer Res 71: 6764-6772, 2011.

36. Kim J, Tang JY, Gong R, Kim J, Lee JJ, Clemons KV, Chong CR, Chang KS, Fereshteh M, Gardner D, et al: Itraconazole, a commonly used antifungal that inhibits Hedgehog pathway activity and cancer growth. Cancer Cell 17: 388-399, 2010.

37. Wang X, Wei S, Zhao Y, Shi C, Liu P, Zhang C, Lei Y, Zhang B, Bai B, Huang Y, et al: Anti-proliferation of breast cancer cells with itraconazole: Hedgehog pathway inhibition induces apoptosis and autophagic cell death. Cancer Lett 385: 128-136, 2017.

38. Liang G, Liu M, Wang Q, Shen Y, Mei H, Li D and Liu W: Itraconazole exerts its anti-melanoma effect by suppressing Hedgehog, Wnt, and PI3K/mTOR signaling pathways. Oncotarget 8: 28510-28525, 2017.

39. Tsubamoto H, Sonoda T, Ikuta S, Tani S, Inoue K and Yamanaka N: Combination chemotherapy with itraconazole for treating metastatic pancreatic cancer in the second-line or additional setting. Anticancer Res 35: 4191-4196, 2015. 